Rapport - Société canadienne d'histoire de l'Église catholique

\title{
Monseigneur Louis-François de Mornay, Capucin, troisième évêque de Québec
}

\section{Gabriel-Marie Dumas}

Volume 30, 1963

URI : https://id.erudit.org/iderudit/1007355ar

DOI : https://doi.org/10.7202/1007355ar

Aller au sommaire du numéro

Éditeur(s)

La Société canadienne d'histoire de l'Église catholique

ISSN

0318-6148 (imprimé)

1927-7075 (numérique)

Découvrir la revue

Citer cet article

Dumas, G.-M. (1963). Monseigneur Louis-François de Mornay, Capucin, troisième évêque de Québec. Rapport - Société canadienne d'histoire de l'Église catholique, 30, 53-61. https://doi.org/10.7202/1007355ar

Tous droits réservés @ La Société canadienne d'histoire de l'Église catholique, 1964
Ce document est protégé par la loi sur le droit d'auteur. L’utilisation des services d'Érudit (y compris la reproduction) est assujettie à sa politique d'utilisation que vous pouvez consulter en ligne.

https://apropos.erudit.org/fr/usagers/politique-dutilisation/ 


\section{Monseigneur \\ Louis-François de Mornay, Capucin, troisième évêque de Québec}

Le 22 juin 1713, le roi Louis XIV nommait $\mathrm{M}^{\mathrm{gr}}$ Louis-François de Mornay, capucin, coadjuteur avec future succession de l'évêché de Québec ${ }^{1}$. Cette nouvelle fit l'objet de nombreux commentaires plus ou moins enthousiastes, dont quelques-uns ne manquent pas d'humour, témoin cette lettre que le supérieur des Missions-Etrangères de Paris, M. Brisacier, écrivait à un confrère : " On nous apprit, il y a quelques jours, dit-il, que la Cour donne à $\mathbf{M}^{\mathrm{gr}}$ de Québec pour Coadjuteur un Capucin, nommé le R.P. De Mornay, gardien de Meudon, homme de qualité et de vertu comme je le présume, mais enfin capucin, et cela sans nous en dire un seul mot, et sans qu'il soit connu de nous. Dieu saura tirer sa gloire de tout, il faut le bénir en tout temps ${ }^{2}$."

Ces paroles, écrites sans malice, je le veux bien, trahissaient une grande déception et reflétaient assez bien le sentiment du clergé de Paris.

Par contre, le ministre Pontchartrain, chargé au nom du roi de présenter à $\mathrm{M}^{\mathbf{g r}}$ de Saint-Vallier son coadjuteur, le fait en des termes pleins d'éloges : "Le roi vient de vous donner pour Coadjuteur à l'évêché de Québec le R. Père de Mornay, Gardien des Capucins de Meudon. C'est un homme de condition et qui joint à sa naissance une grande piété et un esprit propre pour le gouvernement, ayant été depuis 19 ans revêtu des plus grandes dignités de son ordre; il est dans toutes dispositions à votre égard que vous pouvez désirer de lui et je suis persuadé que le choix que sa Majesté a fait vous fera plaisir ${ }^{3}$."

Or, en cette année $1713, \mathrm{M}^{\mathrm{gr}}$ de Saint-Vallier ne désirait plus de coadjuteur; le roi, de son côté, en voulait un pour l'évêque de Québec. Ne pouvant l'écarter, $\mathbf{M}^{\mathrm{gr}}$ de Saint-Vallier voulut le reléguer aux confins de son immense diocèse, la Louisiane ${ }^{4}$, le seul endroit qu'il n'avait pas visité lui-même. Mais, de loin ou de près, un coadjuteur non choisi, non désiré, et parfaitement inconnu, appelé à exercer son ministère sur le territoire du bouillant $\mathbf{M}^{\mathrm{gr}}$ de Saint-Vallier, c'était presque une gageure. $\mathrm{M}^{\mathrm{gr}}$ de Mornay le savait très bien; il usa alors de diplomatie :

1 Paris, Bibl. Nationale, Nouvelles acquisitions, $n^{\circ} 4135$, fol. 255-256. Voir aussi Arch. des Capucins de Paris, ms 1532, p. 355.

2 Arch. publ. du Canada: Arch. du Sém. des Missions-Etrangères de Paris, vol. 18, fol. 489. p. 229.

3 Arch. Nat. de France : Arch. des Colonies, Série B, vol. 35, $3^{\circ}$ partie,

4 Abbé Auguste Gosselin, L'Eglise du Canada depuis $M^{o r}$ de Laval jusqu'à la Conquête, $2^{\circ}$ partie, $M^{\text {gr }}$ de Mornay, $M^{\text {or }}$ Dosquet, $M^{\text {gr }}$ de Lauberivière, Québec, Laflamme et Proulx, 1912, p. 11. 
pour plaire au roi, il accepta l'épiscopat, et pour ne pas indisposer $\mathbf{M}^{\mathrm{gr}}$ de Saint-Vallier, il resta en France.

Et depuis lors, une sorte de mystère plane sur ce personnage, presque ignoré ou du moins mal connu, de l'histoire de l'Eglise catholique au Canada. La cause en est, semble-t-il, son refus de venir au pays. Mais quelles furent les vraies raisons qui motivèrent sa conduite ? La présente étude veut essayer d'y répondre. Le peu de temps dont je dispose, ce matin, m'oblige à me limiter à ce seul aspect - le plus

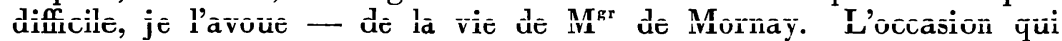
m'est offerte de vous entretenir me donne l'avantage de souligner le trois centième anniversaire de naissance de $\mathrm{M}^{\mathrm{gr}}$ de Mornay et le deux cent cinquantième anniversaire de sa nomination comme coadjuteur de Québec.

Quelques données biographiques sont nécessaires, il me semble, pour bien saisir la suite des faits.

$M^{g r}$ Louis-François de Mornay est né le 12 septembre 1663, non pas à Vannes comme l'ont affirmé tous les historiens en se répétant, mais au château de Du Mesnil-Théribus, près de Beauvais en Normandie $^{5}$. Son père, Charles de Mornay, était de noblesse; il avait perdu une jambe à la célèbre bataille de Rocroy ${ }^{6}$. Monseigneur était le quatrième d'une famille de dix enfants; deux étaient morts en bas àge; les quatre filles devinrent religieuses; trois garçons embrassèrent la carrière des armes comme leur père. Louis-François fit ses études au collège des Jésuites de Vannes ${ }^{7}$ avant d'entrer chez les Capucins de Meudon près de Paris; il fut ordonné prêtre à l'âge de vingt-quatre ans en 1687. Il devint successivement secrétaire provincial, lecteur de théologie, définiteur provincial et supérieur des couvents de Beauvais, de Paris, de Pontoise, de Péronne, d'Ámiens et de Meudon ${ }^{8}$.

C'est à Meudon qu'il prononça en 1711 l'oraison funèbre du grand dauphin, fils de Louis XIV, et l'année suivante celle du jeune dauphin, petit-fils du même roi ${ }^{9}$. Ces deux pièces d'éloquence attirèrent sur lui l'attention du monarque qui le nomma en 1713 évêque d'Eurémie et coadjuteur de Québec avec future succession. Le père de Mornay avait alors quarante-neuf ans. Il reçut ses bulles l'année suivante et fut sacré à Paris dans l'église des Capucins, le 15 avril 1714, par le cardinal de Rohan, évêque de Strasbourg et grand aumônier de France ${ }^{10}$. Il resta coadjuteur jusqu'en 1727, où il succéda à $\mathbf{M}^{\mathrm{gr}}$ de Saint-Vallier, comme évêque de Québec et le demeura jusqu'en 1733; il se démit alors de

5 Acte de baptême extrait du registre de Du Mesnil-Théribus, près de Beauvais.

6 Dictionnaire de Moreri.

7 RochemonteIx, Les Jésuites et la Nouvelle-France au XVIII ${ }^{\bullet}$ siècle, Paris, Picard et Fils, 1906, 2 tomes (cf. tome 1 ${ }^{\text {er }}$, p. 156).

8 Arch. des Capucins de Paris, ms 1532, p. 355.

9 Ibid.

10 Ibid. 
son siège et mourut à Paris, écrasé par une voiture, le 28 novembre 1741, à l'âge de soixante-dix-huit ans.

Pourquoi $\mathrm{M}^{\mathrm{gr}}$ de Mornay, coadjuteur de Québec de 1713 à 1727 puis évêque de 1727 à 1733, ne vint-il pas, pendant ces vingt années, occuper son poste en Nouvelle-France? Certains historiens ont émis les opinions suivantes : la peur de traverser l'océan; son grand âge, sa santé débile; enfin, c'était un évêque de cour. Examinons brièvement ces dites raisons.

$\mathrm{M}^{\mathrm{gr}}$ Henri Têtu, dans son livre Les évêques de Québec, endosse l'opinion de certains quand il écrit : "Quelques-uns disent que ce fut la grande crainte qu'il avait de traverser la mer, qui l'empêcha toujours de venir en Amérique ${ }^{11}$. " Pour sa part, l'abbé Auguste Gosselin, dans son livre $L^{\prime} E$ glise du Canada, écrit dans le même sens : « On a prétendu, dit-il, - et c'est devenu une légende - qu'il ne put jamais se résoudre à traverser la mer : une crainte invincible l'en empêchait ${ }^{12}$. " La peur de l'eau n'était certes pas un motif suffisant pour empêcher le prélat d'accomplir un devoir aussi sacré que l'épiscopat. Il appartenait à une famille de soldats et de héros : son père est blessé à la guerre, ses frères meurent sur les champs de bataille; lui-même, il quitte un château pour entrer chez les Capucins. A son tour, il envoie les Capucins en Louisiane. Ce qu'il demande aux autres, il ne pourrait l'accomplir? Ce serait une lâcheté que contredit d'ailleurs toute sa vie passée. Non, $\mathbf{M}^{\mathrm{gr}}$ de Mornay n'était pas un peureux.

D'autres ont parlé de son grand âge ${ }^{13}$. Cinquante ans était un âge assez avancé pour l'époque évidemment. Mais le roi n'a pas choisi un vieillard pour succéder à $\mathbf{M}^{\text {gr }}$ de Saint-Vallier qui, en 1713, avait soixante-trois ans.

Certains ont mis en cause "sa faible santé ${ }^{14}$ ". Là encore la chose est impensable; peut-on supposer qu'on nomme un malade pour remplacer un évêque âgé et infirme, " seni et valetudinario ${ }^{15}$ "? D'ailleurs celui qui devait mourir à l'âge de soixante-dix-huit ans, écrasé par une voiture, devait jouir d'une santé pour le moins suffisante.

L'abbé Gosselin écrit plus loin dans son même ouvrage : "Sa nomination comme évêque fut une nomination de cour. $\mathbf{M}^{\mathrm{gr}}$ de Mornay appartenait à une noble famille de Bretagne [sic] qui sollicita son élévation à l'épiscopat ${ }^{16}$. " C'est là, je crois, dire beaucoup de choses en peu de mots et surtout sans aucune preuve. $M^{\text {gr }}$ de Mornay ne résida jamais à la cour, mais au couvent des Capucins, et à l'occasion rendit service aux évêques de France et de Belgique pour les confirmations et

$11 \mathrm{M}^{\mathrm{gr}}$ Henri TÊtu, Les évêques de Québec, Québec, Narcisse-S. Hardy, 1889, 692 p. (cf. p. 168).

12 Gosselin, op. cit., p. 10.

13 TACHEREAU, Histoire manuscrite du Séminaire de Québec.

14 Ibid.

15 Rome, Arch. de la Propagande, Acta Camer., n 26, fol. 167.

16 Gosselin, op. cit., p. 3. 
les ordinations. Il fit les fonctions épiscopales durant la vacance du siège de Cambrai après la mort de Fénelon ${ }^{17}$. Evêque de cour, $\mathrm{M}^{\mathrm{gr}}$ de Mornay ne le fut jamais; de plus, son caractère simple, franc et droit l'éloignait de la vie mondaine et courtisane de Versailles.

Enfin, d'autres utilisant un calambour facile ont dit simplement que ce fut un évêque mort-né (Mornay).

Par ailleurs, l'abbé Gosselin a touché vraiment au fond du problème quand il écrit : "Nous croyons surtout que, connaissant le caractère de $\mathbf{M}^{\mathrm{gr}}$ de St-Vallier qui aimait à gouverner par lui-même, connaissant surtout les difficultés qu'il avait avec son Séminaire de Québec, celles qu'il avait eues également avec les religieux, dans le passé, il $\left[\mathrm{M}^{\mathrm{gr}}\right.$ de Mornay] pensa qu'il valait mieux pour lui rester en dehors de ces querelles et laisser le prélat administrer son diocèse à sa guise. Il resta donc à Paris ${ }^{18}$. »

Il faut faire remarquer que $\mathrm{M}^{\mathrm{gr}}$ de Mornay était coadjuteur de l'évêché de Québec, et non pas auxiliaire de l'évêque de Québec. Cette distinction a son importance; l'auxiliaire est nommé pour aider l'évêque résidentiel et il ne peut s'y dérober du moment qu'il a accepté cette charge. L'évêque coadjuteur avec future succession est nommé pour assurer la continuité du siège épiscopal; il jouit d'une grande indépendance vis-à-vis de l'évêque résidentiel à qui il doit succéder automatiquement.

"De plus, au commencement du XVIII ${ }^{\mathrm{e}}$ siècle, un évêque suffisait au diocèse de Québec. Malgré l'étendue des terres de sa juridiction, sa population ne dépassait pas cinquante mille. Ce seul évêque, avec des vicaires généraux dans les missions éloignées, pouvait répondre aux exigences du ministère des âmes. C'est bien ce que pensait $\mathrm{M}^{\mathrm{gr}}$ de St-Vallier. Il ne désirait pas de coadjuteur. Il en avait demandé un lorsqu'il était retenu en Angleterre et en France, c'est-à-dire pendant treize ans, de 1700 à $1713^{19}$." Il aurait alors accepté un Sulpicien : "Parce que, écrit-il, l'esprit de ménagement et de douceur que l'on remarque ordinairement dans ceux qui sont élevés dans cette maison, les rend plus propres que d'autres à ce diocèse ${ }^{20}$. 》

17 Arch. des Capucins de Paris, ms 1532, p. 355. « ${ }^{\mathrm{gr}}$ l'Archevêque de Cambrai, M. de Fénélon, étant décédé, il y fut envoyé dans la Semaine Sainte 1716, pour y donner les ordres et y faire les fonctions épiscopales, d'où il ne retourna à Paris, à St-Honoré, que sur la fin de juillet. s

- D'après l'abbé Brasseur de Bourbourg : la mort de Louis XIV qui arriva un an après le sacre de $\mathbf{M}^{\mathrm{gr}}$ de Mornay fit naître sans doute des circonstances qui l'empêchèrent de se rendre à Québec. Fénélon étant mort dans le même temps, l'évêque d'Euménie fut chargé de l'administration de l'église de Cambrai pendant plusieurs années ": cf. lettre de l'abbé Louis Cesbron-Lavau, 5, rue Chevreul, Angers (Maine-et-Loire).

18 Gosselin, op. cit., p. 10.

19 Père PlaCide, o.f.m.cap., Essai biographique de $M^{\text {gr }}$ de Mornay, Capucin, troisième évêque de Québec, dans la revue Charité (à l'usage des Capucins de la province de l'Est du Canada), oct.-nov. 1959, p. 47.

20 Gosselin, op. cit., p. 4. 
C'était bien cet esprit de ménagement et de douceur qui manquait au deuxième évêque de Québec. $\mathbf{M}^{\mathrm{gr}}$ de Saint-Vallier était un saint homme, mais d'un caractère impossible. Vous me permettrez de vous le décrire un peu; cela vous expliquera pourquoi le roi voulait s'en défaire et nommer un homme plus traitable à sa place.

Laissons l'abbé Gosselin nous le présenter : «Il avait, dit-il, un caractère difficile, autoritaire, peu accessible à la contradiction ${ }^{21}$. " «... Pour être ami de $\mathrm{M}^{\mathrm{gr}}$ de St-Vallier, il fallait l'approuver en tout et ne le contredire en rien ${ }^{22}$... Il n'était pas homme à faire faire son travail par les autres : il agissait lui-même, et il le faisait avec l'impétuosité et l'ardeur de sa nature ${ }^{23}$. " "Tout âgé qu'il est, c'est encore lui, et lui seul, qui gouverne son diocèse; c'est lui qui voit à tout, qui conduit tout ${ }^{24}$." M. Faillon, sulpicien, déclare à son tour : "Ce prélat, contre la pratique générale des évêques, avait agi jusqu'alors sans conseil dans son administration, quoiqu'il eût peut-être plus besoin que d'autres de conseils sages et prudents, à cause de la promptitude de son caractère ${ }^{25}$.

Ces textes montrent bien jusqu'à quel point l'évêque ne voulait pas de collaborateurs. D'ailleurs, il le fit bien voir après la démission de $\mathrm{M}^{\mathrm{gr}}$ de Laval en 1688. Il essaya d'empêcher le saint fondateur de revenir à Québec pour terminer ses jours; n'ayant pas réussi, il le relégua à Saint-Joachim-du-Cap-Tourmente ${ }^{26}$. De son exil, $\mathbf{M}^{\mathrm{gr}}$ de Laval disait : "Ce m'est une douleur bien sensible de voir que celui qui devrait être l'appui de cette Eglise naissante serve d'instrument au démon pour travailler à sa destruction, tâchant d'y mettre la division et la confusion ${ }^{27}$,

Ces dernières paroles se réalisèrent rapidement : "A la fin de 1694, écrit l'abbé Gosselin, l'évêque de Québec s'était aliéné presque tous les esprits au Canada; on le regarde comme un homme impossible ${ }^{28}$. " Il était entré en conflit avec le Séminaire et, par la suite, avec la majorité du clergé tant séculier que régulier. Il s'était brouillé avec le gouverneur Frontenac, avec l'intendant Champigny, avec des officiers de l'armée et de l'administration comme Callières et Ramesay ${ }^{29}$, à tel point que le ministre Pontchartrain et l'archevêque de Paris lui signifièrent de se présenter au roi pour rendre compte de sa conduite ${ }^{30}$.

21 Abbé Auguste Gosselin, L'Eglise du Canada, première partie, $M^{g r}$ de Saint-Vallier, Québec, Laflamme et Proulx, 1911, 503 p. (cf. p. 210).

22 Id., p. 322.

23 Id., p. 58.

24 Id., p. 434.

25 Id., p. 246.

26 Abbé Auguste Gosselin, Vie de $M^{o r}$ de Laval, premier évêque de Québec et apôtre du Canada, 1622-1708, Québec, L.-J. Demers et Frère, 1890, 2 tomes (cf. tome 2, p. 406).

${ }_{27}$ Rev. de l'Univ. Laval, oct. 1954, p. 96.

28 Gosselin, Eglise du Canada, $2^{\mathrm{e}}$ partie, p. 108.

29 Id., p. 117.

30 Id., p. 130. 
Son départ fut un soulagement pour tous, et quand le ministre voulut obtenir la renonciation à son évêché, peu de gens, s'il y en eut ${ }^{31}$, plaidèrent en sa faveur, en tout cas pas les Jésuites qui souhaitaient le changement d'évêque ${ }^{32}$. $\quad M^{\text {gr }}$ de Laval disait de lui en 1690 : "Son retour est le plus grand mal qui puisse arriver à cette Eglise ${ }^{33}$."

Pour la même raison, l'évêque dut retourner en France en 1700, à la demande du roi qui le retint quatre ans, essayant en vain de le faire démissionner. $\mathrm{M}^{\mathrm{gr}}$ de Saint-Vallier réussit enfin à s'embarquer en

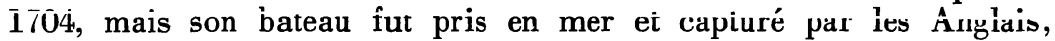
l'évêque séjourna quatre ans comme prisonnier en Angleterre. De retour en France, le roi le garda encore quatre ans sans pouvoir obtenir sa démission. Enfin, le ministre Pontchartrain lui proposa au moins trois solutions honorables de plaire au monarque : la première, donner la démission de son évêché à des conditions honnêtes; la deuxième, s'en retourner présentement dans son diocèse, promettant de donner sa démission dans peu d'années; la troisième, aller cette année dans son diocèse, en demandant auparavant un coadjuteur qui sera accordé, et qui se rendra le plus tôt qu'il pourra les années suivantes; sur cela, faire promesse de revenir dans quelques années donner sa démission ${ }^{34}$.

La dernière proposition fut acceptée en partie. L'évêque de Québec s'empressa de partir pour son lointain diocèse et Louis XIV nomma un coadjuteur qu'il mit au courant de la situation religieuse de la NouvelleFrance. Mais $\mathrm{M}^{\mathrm{gr}}$ de Saint-Vallier, une fois rendu au Canada, ne voulut jamais démissionner. Aussi le coadjuteur attendit-il pendant quatorze ans cette démission qui ne vint pas. Il resta à Paris. Cependant, à bout de patience et d'espérance, il offrit au roi sa démission en mars 1728. Or, à cette date, il était devenu, sans le savoir et malgré lui, évêque de Québec, car son prédécesseur était mort le 26 décembre 1727. Sa démission ne valait donc pas et n'eut pas de suite.

$\mathrm{M}^{\mathrm{gr}}$ de Mornay envoya au chanoine Eustache Chartier de Lotbinière une procuration à l'effet de prendre possession, en son nom, du siège épiscopal. Cet acte occasionna, entre le délégué et les autres chanoines ${ }^{35}$, une vive opposition qui est demeurée tristement célèbre comme d'ailleurs la sépulture du deuxième évêque de Québec ${ }^{36}$. La vie orageuse de $\mathrm{M}^{\mathrm{gr}}$ de Saint-Vallier ne pouvait que se terminer dans la tempête et l'orage.

$31 \quad I d .$, p. 108.

32 Id., p. 127. « On sait que le Prélat $\left[\mathrm{M}^{\mathrm{gr}}\right.$ de Saint-Vallier $]$ avait fort mécontenté la Compagnie en retranchant, en faveur de la paroisse, certains catéchismes et offices religieux qui se faisaient dans leur église : . Nous espérons, dit le père Claude Chauchetière, le rétablissement de tous nos emplois, peut-être par le changement d'évêque... *

33 Gosselin, Vie de $M^{\text {gr }}$ de Laval, tome 2, p. 448.

$34 M^{g r}$ de Saint-Vallier et l'Hôpital Général de Québec, Québec, G. Darveau, 1882 (cf. p. 216-220).

35 Gosselin, Eglise du Canada, $2^{\circ}$ partie, p. 30.

36 Gosselin, Eglise du Canada, ${ }^{\mathrm{re}}$ partie, p. $452 \mathrm{~s}$. 
$M^{\text {gr }}$ de Mornay s'était toujours tenu au courant des affaires du diocèse de Québec. Ces dernières difficultés ne l'incitaient pas beaucoup à se rendre au Canada. Eut-il alors l'idée de démissionner, comme on l'a affirmé ${ }^{37}$ ? Aucun document de l'époque ne le mentionne. En tout cas, $\mathrm{M}^{\mathrm{gr}}$ de Mornay crut bien faire en se choisissant un coadjuteur qui irait le plus tôt possible, puisqu'il était déjà sacré évêque - il s'agit de $\mathrm{M}^{\mathrm{gr}}$ Dosquet - pour régler les problèmes urgents. Puis il écrivit aux chanoines pour leur apprendre cette nouvelle et les inviter surtout à tenir une conduite qui convenait mieux à leur dignité et à leur âge. Cette lettre, que je cite, est celle d'un parfait gentilhomme :

$$
\text { Paris, le } 20 \text { mai } 1729 .
$$

Je ne répondrai pas, Messieurs, aux articles particuliers de la lettre que vous m'avez fait l'honneur de m'écrire. La paix et la charité ne consistent pas dans les disputes. Il faut en éviter toutes les occasions. Tenez-vous-en à ce que monseigneur l'évêque de Samos, mon coadjuteur, vous dira de ma part. C'est lui qui est aujourd'hui revêtu de tous mes pouvoirs et en ce cas même qu'il vint à mourir (ce qu'à Dieu ne plaise) je vous déclare que le gouvernement du diocèse sera entièrement dévolu à celui ou à ceux qu'il aura établi grand vicaire ou vicaire général, selon le pouvoir que je lui en ai donné, et qu'on ne pourra rien changer sans des ordres exprès et positifs de ma part ${ }^{38}$.

L'abbé Gosselin commente ainsi ce texte : "Cette lettre annonçait un homme de nerf, tel qu'en avait besoin l'Eglise de la Nouvelle-France dans les circonstances fâcheuses où elle se trouvait. Malheureusement le troisième évêque de Québec ne put jamais se résoudre à venir au Canada ${ }^{39}$.

Nous avons déjà indiqué les motifs qui ont empêché $\mathrm{M}^{\mathrm{gr}}$ de Mornay de se rendre en Nouvelle-France pendant qu'il était coadjuteur. Mais pourquoi, une fois devenu évêque, ne vint-il pas occuper son siège?

Il faut dire d'abord qu'en $1728, \mathrm{M}^{\mathrm{gr}}$ de Mornay avait soixante-cinq ans; l'âge même de $\mathrm{M}^{\mathrm{gr}}$ de Laval lorsqu'il démissionna en 1688. La raison de sa vieillesse et de sa mauvaise santé fut donc le principal obstacle qui s'opposa à sa venue à Québec. C'est d'ailleurs la raison qu'il fit valoir à la Cour pour demander un coadjuteur comme nous l'apprend une lettre du roi au gouverneur Beauharnais : "L'évêque de Québec, ne pouvant, à cause de son grand âge et de ses infirmités, se rendre au Canada, je lui ai donné pour coadjuteur le sieur Dosquet, évêque de Samos, qui a déjà passé quelques années dans la colonie, alors qu'il était simple prêtre ${ }^{40}$.

Malgré cette nomination, le troisième évêque de Québec gardait encore l'espoir de venir au pays comme nous le montre une lettre de M. Tremblay, des Missions-Etrangères de Paris : "M. Delorme, dit-il, nous avait assuré que $\mathrm{M}^{\mathrm{gr}}$ de Mornay avait fait sa démission de la

37 Bull. Rech. Hist., IV, p. 264.

38 Gosselin, Eglise du Canada, $1^{\text {re }}$ partie, p. 472.

39 Ibid.

40 Gosselin, Eglise du Canada, $2^{\bullet}$ partie, p. 36. 
coadjutorerie, cependant nous apprenons par ce qu'il a dit à M. Formel qu'il est tout prêt d'aller en Canada et disposé à y passer par les vaisseaux qui [partiront bientôt]... et vous aidera à pacifier tous les troubles et pourra peut-être vous servir à redonner au Séminaire ce dont il a joui jusqu'à ce que l'évêque défunt le lui ait ôté ${ }^{41}$. »

De plus, il était désiré au Canada selon le témoignage du chanoine Hazeur de l'Orme : " J'ai raconté à $\mathbf{M}^{\text {gr }}$ de Mornay, dit-il, une bonne partie de ce qui se passe en Canada... [et] M. de Noyan lui a raconté plusieurs traits de $\mathbb{N}^{3 *}{ }^{*}$ Dosquet, et lui a fair entendre que le Canada le souhaitait avec empressement. Cela a paru lui faire d'autant plus de plaisir qu'il n'est pas content de la manière dont $\mathbf{M}^{\mathrm{gr}}$ Dosquet a agi envers M. de Falaize ${ }^{42}$.»

Le désir de $\mathrm{M}^{\mathrm{gr}}$ de Mornay de se rendre au pays était bien légitime. La population du Canada aurait été fort contente de le connaître et d'apprécier surtout ses belles qualités de sagesse et de paix, de prudence et de pondération qui ont marqué son action dans les charges et qui auraient été si nécessaires en ces années troublées de 1728. L'historien Antoine Bernard, c.s.v., émet cette opinion qui me paraît fort judicieuse : "Il hésita, semble-t-il, devant l'inextricable écheveau des complications politico-religieuses de son époque. Il n'avait pas le tempérament " batailleur " qui permet de vivre parmi les fauves ${ }^{43}$."

Il laissa cette tâche à $\mathbf{M}^{\mathrm{gr}}$ Dosquet qui n'a pas réussi; quant à lui, il s'employa avec succès à gouverner cette partie lointaine de son diocèse, la Louisiane. Ici, comme à Québec, les conflits de juridiction, les ingérences religieuses ne manquaient pas, mais la fermeté de $\mathbf{M}^{\mathrm{gr}}$ de Mornay en viendra à bout. Pour ce faire, il dut s'opposer souvent au Conseil de la Compagnie des Indes Occidentales, au cardinal de Fleury et même au ministre, le comte de Maurepas. Je cite ici, comme exemple, la conclusion d'une lettre que $\mathrm{M}^{\mathrm{gr}}$ de Mornay adressait à ce dernier, le 14 novembre 1731 : "Vous ne devez pas douter de ma soumission aux ordres du roi, mais je crois devoir vous représenter ce que j'ai fait pour remplir le ministère que Dieu m'a confié, auquel seul nous en devons répondre et que le roi a la bonté de ne pas troubler ${ }^{44}$."

C'est vraiment dommage qu'un évêque de la trempe de $\mathrm{M}^{\mathrm{gr}}$ de Mornay n'ait pas pu venir au Canada. Quand sa succession fut assurée et que les difficultés de la Louisiane furent applanies, il démissionna du siège de Québec en faveur de $\mathrm{M}^{\mathrm{gr}}$ Dosquet, son coadjuteur, le 12 septembre 1733 , le jour même de sa fête de naissance : il avait soixantedix ans. En décembre suivant, il fut pourvu du prieuré d'Arbois, ordre de Citeaux, au diocèse de Besançon. En 1736, il eut le bonheur de consacrer $\mathrm{M}^{\mathrm{gr}}$ de Lauberivière, son deuxième successeur immédiat ${ }^{45}$.

41 Arch. du Sém. de Québec, Lettres $M, \mathrm{n}^{\circ} 60$.

42 Arch. du Sém. de Québec, cahier $n^{\circ} 208$.

43 Lettre que le frère Bernard m'a écrite en mars 1963.

44 Arch. du Sém. de Québec, Evếques, no 171c.

45 Gosselin, Eglise du Canada, $2^{\mathrm{e}}$ partie, p. 361. 
Il vécut encore huit ans à Paris, chez les Capucins, jusqu'à sa mort survenue le 28 novembre 1741. Il traversait la rue Saint-Honoré en face du couvent, lorsqu'il fut écrasé par un carosse tiré par quatre chevaux. Il mourut sur-le-champ; il avait soixante-dix-huit ans.

La fin tragique de $\mathrm{M}^{\mathrm{gr}}$ de Mornay fut signalée à la Cour et dans les journaux de Paris ${ }^{46}$. Mais, en Nouvelle-France, elle passa presque inaperçue, comme d'ailleurs son épiscopat qui dura vingt-huit ans. Cet homme, qui présentait de belles qualités de distinction, de sagesse, d'humilité et de force d'âme, avait été choisi par Louis XIV pour apporter la paix dans le diocèse de Québec que $\mathbf{M}^{\mathrm{gr}}$ de Saint-Vallier dirigeait dans la crainte, sinon dans l'amour. Tout pliait devant l'autorité de ce dernier, car l'opposition s'effaçait nécessairement devant les interdits, les suspenses, les excommunications, les retraits de juridiction, les admonitions aux gouverneurs, au conseil souverain, les monitions canoniques aux religieux, la déposition des supérieures de communautés.

$\mathrm{M}^{\mathrm{gr}}$ de Mornay, nommé coadjuteur, a cru en conscience que, devant ces faits connus en France et au Canada, il ne pouvait jouer son rôle pacificateur, tant que l'évêque résidentiel n'aurait pas démissionné, conformément aux intentions du roi. Mais Louis XIV mourait en 1715 et, après lui, la Cour de Versailles se désintéressa des affaires religieuses du Canada. $M^{\text {gr }}$ de Mornay pensa alors qu'il devait sacrifier sa vie d'évêque. Mieux valait laisser les choses dans un état qui semblait permis par la divine Providence ${ }^{47}$.

Son effacement a été mal interprété par certains historiens. Ils ont cru discerner, dans ce geste, une faiblesse, voire une désertion au profit d'une vie plus facile à Paris. Quant à nous, nous pensons que ce fut le désir de laisser intacte l'autorité ombrageuse du deuxième évêque de Québec et d'éviter les conflits possibles qui retint $\mathbf{M}^{\mathbf{g r}}$ de Mornay en France. Pourquoi venir ajouter à la tension des esprits? Dans un recul de deux siècles et demi, son sacrifice n'en paraît que plus grand et plus désintéressé. Le bien des âmes et de l'Eglise a prévalu sur ses intérêts personnels qu'il aurait pu assurer facilement. Mais comme il l'a déjà dit « la paix et la charité ne se trouvent pas dans les disputes ${ }^{48}$ ".

C'est ce que nous avons essayé de démontrer dans cette étude. Puisse-t-elle avoir contribué à faire apprécier, sous son vrai jour, la noble figure de $\mathrm{M}^{\mathrm{gr}}$ de Mornay et, si possible, je l'espère, rendre à ce digne prélat l'estime et le respect auxquels il a droit.

Père Gabriel-Marie Dumas, o.f.m.cap., L. ès L., M.A., Séminaire Saint-François, Cap-Rouge, Qué.

46 Arch. Sém. de Québec, Fonds Verreau, bte 31, liasse 5, $\mathrm{n}^{\circ} 30$, carton 71.

47 Père Placide, op. cit., p. 57.

48 Gosserin, Eglise du Canada, $1^{\text {re }}$ partie, p. 472. 